\title{
Empty Sella Syndrome: A Case Report and Literature Review
}

\author{
Kiran Kumar1*, Mohammed Khalid1, Alyaa Fadhil2, Pankaj Lamba3 ${ }^{3}$, Shaikh Altaf Basha1, \\ Salwa Abd El Zaher Mabrouk Ibrahim ${ }^{1}$ \\ ${ }^{1}$ Department of Internal Medicine, GMCH \& RC, Ajman, UAE \\ ${ }^{2}$ Department of Clinical Pharmacology, GMCH \& RC, Ajman, UAE \\ ${ }^{3}$ Department of Ophthalmology, GMCH \& RC, Ajman, UAE \\ Email: ${ }^{*}$ drkiran k@yahoo.com
}

Received 7 March 2015; accepted 21 March 2015; published 25 March 2015

Copyright (C) 2015 by authors and Scientific Research Publishing Inc.

This work is licensed under the Creative Commons Attribution International License (CC BY).

http://creativecommons.org/licenses/by/4.0/

(c) () Open Access

\begin{abstract}
An empty sella occurs due to herniation of the arachnoid through an incompetent diaphragma sellae. Over time, cerebrospinal fluid (CSF) pulsations may enlarge the sella and compress the gland against the floor of the sella. Empty sella syndrome is considered as a less common entity and is usually asymptomatic and an incidental finding. However, it can be a manifestation of increased intracranial pressure and can be occasionally severe. Compression of the pituitary gland may affect function, or traction on the optic chiasm and nerves may cause visual symptoms. An empty sella may be classified as primary when this occurs in persons who have not received pituitary radiation or pituitary surgery, while an empty sella discovered following such procedures is classified as secondary empty sella. In this report, we presented a 41-year-old multiparous patient who presented to us with symptoms of headache and left sided hemi-sensory disturbance. Examination was unrevealing except for the fact that she was obese. On evaluation, she was detected to have impaired blood sugars, dyslipidemia and vitamin insufficiency. MRI brain revealed presence of Empty Sella. Further hormonal analysis was normal. She was treated conservatively and regular follow-up was advised.
\end{abstract}

\section{Keywords}

Empty Sella Syndrome, Empty Sella, Pituitary Dysfunction

\section{Introduction}

Empty sella syndrome (ESS) is a condition in which the sella turcica is partially or completely filled with CSF.

"Corresponding author.

How to cite this paper: Kumar, K., Khalid, M., Fadhil, A., Lamba, P., Basha, S.A. and El Zaher Mabrouk Ibrahim, S.A. (2015) Empty Sella Syndrome: A Case Report and Literature Review. Neuroscience \& Medicine, 6, 42-45. 
This results in compression and displacement of normal pituitary gland [1]. It can be either primary or secondary. Two factors play a role in primary empty sella. One is an inherent weakness of the diaphragm sella, and second is an increase in the intracranial pressure which promotes the herniation of arachnoid membrane into the pituitary fossa [2]. Primary empty sella syndrome is an incidental radiological diagnosis and usually asymptomatic. It is more common in middle-aged obese females. They present with headache and are occasionally associated with endocrine or visual abnormalities [3]. Secondary empty sella occurs in the background of previous surgery or irradiation. It can also be due to hemorrhage or infarction of pituitary gland. In this report we presented a patient who presented to us with left sided hemisensory disturbance and headache and had an associated incidental finding of an empty sella syndrome.

\section{Case Report}

A 41-year-old obese multiparous lady presented to us with numbness of left side of the body (face, left upper limb and left lower limb) since 6 years. Symptoms are intermittent and are associated with intermittent headache and neck pain. She also gives history of left facial weakness six years ago which recovered gradually. There is no weakness of extremities or imbalance. She also complained of visual blurring of recent onset. Her menstrual history was normal. On examination, her blood pressure was $140 / 80 \mathrm{~mm}$ of $\mathrm{Hg}$ and heart rate was 80 beats per minute. Cardiovascular and respiratory system examination was normal. Central nervous system examination was unremarkable too. Patient had undergone MRI of the cervical spine and was told to have cervical disc disease and was under regular physiotherapy for the same.

An MRI brain was done which revealed presence of few lacunar infarcts in the high parietal area and also an incidental empty sella (Figure 1 and Figure 2).

An ophthalmologic evaluation revealed normal vision with mild bilateral optic disc edema (Figure 3 and Figure 4).

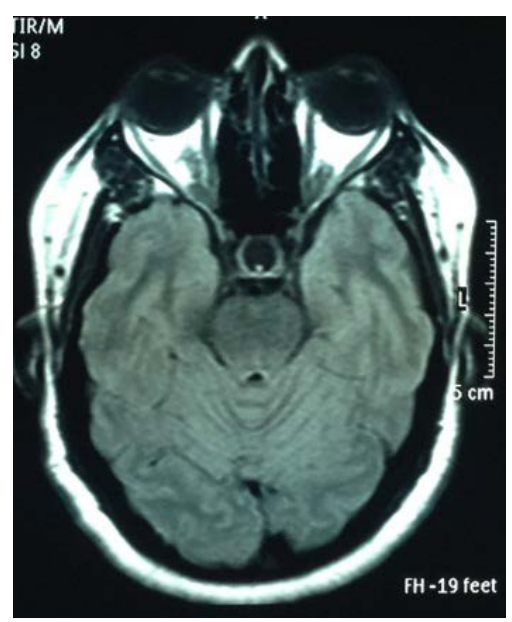

Figure 1. MRI brain (T2 FLAIR-axial) showing empty sella.

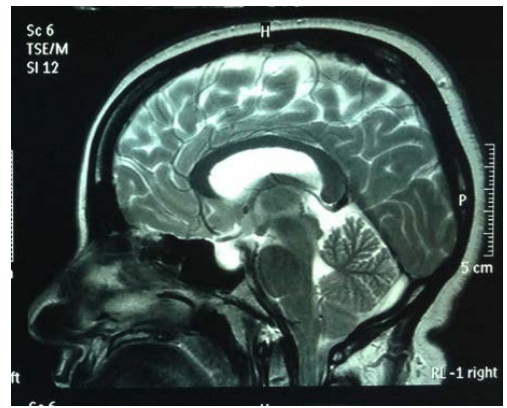

Figure 2. MRI brian (T2 saggital view) showing hyperintense signal intensity in the region of sella turcica suggestive of CSF filled sella. 


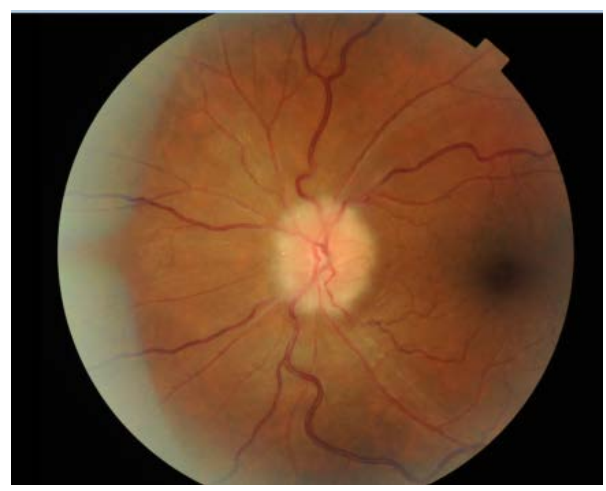

Figure 3. Fundscopy image showing optic disc edema in the left eye.

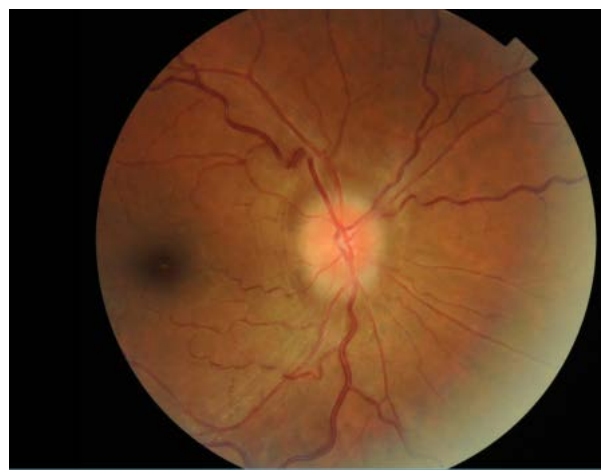

Figure 4. Fundscopy image showing optic disc edema in the right eye.

In view of the finding of empty sella, pituitary hormonal studies were recommended. The TSH was $1.18 \mathrm{mi}-$ cro IU/mL (normal range: 0.27 - 4.2), prolactin levels was 128.9 micro IU/mL (normal range: 102 - 496), follicular stimulating hormone level was 10.06 micro $\mathrm{IU} / \mathrm{mL}$ and lutenising hormone (LH) level was 3.59 micro $\mathrm{IU} / \mathrm{mL}$. All were within normal limits. Serum 25-OH vitamin D level was $13.8 \mathrm{ng} / \mathrm{mL}$ (insufficient: 10 - 29). She was also detected to have impaired post-prandial blood glucose and dyslipidaemia.

She was started treatment with vitamin D replacement, calcium supplements, statins and pregabalin. On follow up after 3 months, patient was symptomatically better. The left sided numbness had reduced and vision was also normal. She has been advised to continue the same treatment and to undergo follow-up MRI brain after 6 months.

\section{Discussion}

The term empty sella syndrome describes a distinct radiological and anatomical entity. The subarachnoid space extends significantly through an incompetent diaphragm sella into the sella turcica [4]. It is well known that the term Empty Sella is incorrect, because in these cases the sella is not empty. In fact, it is completely filled by the pituitary gland, with its stalk, the arachnoid, the CSF and occasionally, the optic system and the third ventricle. That is why intra-sellarar achnoidocele is the preferred term because it expresses in a simple and clear way the findings in this entity [2]. It occurs more commonly in middle aged females and is associated with several conditions such as obesity, hypertension, and migraine [5]. The most reasonable explanation for development of primary empty sella is that the condition arises in a patient who has either a transient or constant elevation in intracranial pressure and who has incompetent diaphragm sella that allows the subarachnoid space to be forced into the sella by the hydrostatic pressure and pulsatile movement of CSF [6]. Secondary ESS is generally associated with a previous surgery, radiotherapy, or medical treatment for tumors of the sellar region [1].

In a recent study, Ghatnatti et al. [7] reported that PES accounted for most cases of empty sella and it was more commonly noted in females with higher parity like in our patient. Enlargement of the pituitary during pregnancy may lead to weakening of the sellar diaphragm, thus predisposing to herniation of cerebrospinal fluid into 
the sella [8]. They also noted that obesity was more common among patients with PES. Obesity causes obstructive sleep apnea leading to hypercapnia and increased CSF pressure predisposing to empty sella. This could also be the contributory cause in our patient.

There is a wide variation in the reported prevalence of endocrine abnormalities in PES. Ghatnatti et al. [7] noted endocrine dysfunction in 50\% of PES patients while De Marinis et al. [8] found endocrine abnormalities in $19 \%$. Hyperprolactinemia was the most common endocrine abnormality observed. In PES particularly, mild hyperprolactinemia has been frequently reported. This is thought to be due to pituitary stalk compression as a consequence of the remodeling of the hypothalamo-pituitary region and altered CSF dynamics.

Surgical indications for correction of symptomatic ESS are controversial and are rare. Visual disturbances and CSF rhinorrhea are the main indications for surgery. When surgery is indicated, the type of surgery depends on clinical presentation and radiological findings. The surgical outcome of cases with ESS is favorable, as most patients report improvement or stabilization of their symptoms [9].

Our patient had an incidentally detected empty sella syndrome during evaluation of headache. Her symptom of left sided numbness was likely due to her cervical disc disease. Her hormonal assays were normal. Visual blurring could likely be due to stretch on the optic tracts. She however, recovered with conservative treatment and did not require any intervention. The most likely cause of empty sella in our patient is her multi-parity and obesity; two factors which are closely linked with the pathogenesis of empty sella syndrome [7] [8].

\section{Conclusion}

Emtpy sella syndrome is an incidental finding which arises in a patient who has either a transient or constant elevation in intracranial pressure and who has incompetent diaphragm sella that allows the subarachnoid space to be forced into the sella by the hydrostatic pressure and pulsatile movement of CSF. It is usually seen in obese patients with slight female preponderance. It should be kept as a differential diagnosis for patients presenting with non-specific headache especially in obese multiparous females.

\section{References}

[1] Braatvedt, G.D. and Corral, R.J. (1992) The Empty Sella Syndrome: Much Do about Nothing. British Journal of Hospital Medicine, 47, 523-525.

[2] Sander, E.C. and Peter Jr., W.C. (1996) Empty Sella Syndrome. In: Wilkins, R. and Rengachery, S., Eds., Neurosurgery, McGraw-Hill, New York, 1367-1373.

[3] Olson, D.R., Guiot, G. and Dereme, P. (1972) The Symptomatic Empty Sella: Prevention and Correction via the Transsphenoidal Approach. Journal of Neurosurgery, 37, 533-537. http://dx.doi.org/10.3171/jns.1972.37.5.0533

[4] Arlot, S., Lalau, J.D., Galibert, P., et al. (1985) Primary Empty Sella Turcica. Analysis of 14 Cases and Review of Literature. Ann Endocrinol (Paris), 46, 99-105.

[5] Degli, U.E.C., Teodori, V., Trasforini, G., et al. (1989) The Empty Sella Syndrome. Clinical, Radiological and Endocrinologic Analysis in 20 Cases. Minerva Endocrinologica, 14, 1-18.

[6] Bragagni, G., Bianconcini, G., Mazzali, F., et al. (1995) 43 Cases of Primary Empty Sella Syndrome: A Case Series. Ann Ital Med Int, 10, 138-142.

[7] Ghatnatti, V., Sarma, D. and Saikia, U. (2012) Empty Sella Syndrome-Beyond Being an Incidental Finding. Indian Journal of Endocrinology and Metabolism, 16, S321-S323.

[8] De Marinis, L., Bonadonna, S., Bianchi, A., Maira, G. and Giustina, A. (2005) Primary Empty Sella. Journal of Clinical Endocrinology \& Metabolism, 90, 5471-5477. http://dx.doi.org/10.1210/jc.2005-0288

[9] Fouad, W. (2011) Review of Empty Sella Syndrome and Its Surgical Management. Alexandria Journal of Medicine, 47, 139-147. http://dx.doi.org/10.1016/j.ajme.2011.06.005 